\title{
Article \\ Through-Floor Vital Sign Searching for Trapped Person Using Wireless-Netted UWB Radars
}

\author{
Zhenghuan Xia ${ }^{1} \mathbb{D}$, Shiyou Wu ${ }^{2}$, Xin Liu ${ }^{1, *}$, Huifeng Shi ${ }^{1}$, Shichao Jin ${ }^{1}$ and Tao Peng ${ }^{1}$ \\ 1 State Key Laboratory of Space-Ground Integrated Information Technology, Beijing Institute of Satellite \\ Information Engineering, Beijing 100095, China; maxwell_xia@126.com (Z.X.); shi-hf@163.com (H.S.); \\ jinsc@163.com (S.J.); pengt2021@126.com (T.P.) \\ 2 Key Laboratory of Electromagnetic Radiation and Sensing Technology, Aerospace Information Research \\ Institute, Chinese Academy of Sciences, Beijing 100190, China; ahwushiyou@126.com \\ * Correspondence: liuxin115@foxmail.com
}

Citation: Xia, Z.; Wu, S.; Liu, X.; Shi, H.; Jin, S.; Peng, T. Through-Floor Vital Sign Searching for Trapped Person Using Wireless-Netted UWB Radars. Appl. Sci. 2021, 11, 10538. https://doi.org/10.3390/app112210538

Academic Editor: Amerigo Capria

Received: 7 October 2021

Accepted: 5 November 2021

Published: 9 November 2021

Publisher's Note: MDPI stays neutral with regard to jurisdictional claims in published maps and institutional affiliations.

Copyright: (c) 2021 by the authors. Licensee MDPI, Basel, Switzerland. This article is an open access article distributed under the terms and conditions of the Creative Commons Attribution (CC BY) license (https:// creativecommons.org/licenses/by/ $4.0 /)$.

\begin{abstract}
In personnel rescue applications, considerable attention has been paid to the positioning method of living persons. Especially, the effective vital sign searching is urgently required. This work presents an effective multiple-observation-points-based vital sign searching method for trapped persons using wireless-netted ultra-wideband (UWB) radars. Controlled by a wireless network, four UWB radars are connected to form a group. Based on that, we design an optimized searching path strategy and the corresponding vital sign searching procedure. The simulated result illustrates the proposed vital sign searching strategy on the spatial locations of the trapped person. Finally, the proof-of-principle through-floor experiment was also carried out, verifying the performance of this proposed vital sign searching algorithm.
\end{abstract}

Keywords: vital sign searching; through-floor; trapped person; wireless-netted radar; UWB radar

\section{Introduction}

In the personnel rescue scenarios, personnel positioning methods have attracted extensive interest in scientific research and engineering applications. In this context, ultrawideband (UWB) radar can detect the vital signs caused by breathing or micro movements of people under ruins, which plays an important role in detecting trapped living persons and shows significant advantages in strong anti-interference ability, high penetrability, and range resolution.

Currently, the period estimation for respiration or similar vital signs, the removal of the stationary/nonstationary clutter, and radar system design [1-7] have lately received great attention for the detection and rescue of life signal by the UWB radar. For the radar system, the more observation points, the greater the possibility for finding the trapped person. If no vital signs are detected at a fixed observation point, selecting subsequent observations can increase the probability of detection. However, vital sign search methods based on different observation points are facing challenges and it is worthwhile devoting much effort to this. In this way, rescuers can thoroughly analyze radar echoes at multiple observation points to extract hidden vital signs of trapped persons [8-12]. Hence, the vital sign searching strategy based on different observation points has emerged as an interesting candidate because of its impressive ability to detect trapped persons in some complex scenes, such as through walls.

Further, the multiview and netted radar systems were found to improve the signalto-noise ratio (SNR) and increase rescue efficiency in $[13,14]$. The development of UWB radar vital sign detection techniques that allow multipoint observation and data association processing is becoming a priority, motivating the design of multiobservation point detection and decision systems that increase rescue efficiency.

In this paper, a novel multiple-observation-points-based vital sign searching strategy for rescuing trapped persons is introduced. Controlled by a wireless network, four UWB 
life-detection radars are set up on a 2D observation aperture and connected to form a group. The main contribution of this paper is the multiple-observation-points-based vital sign searching strategy, including the design of the searching path and decision procedure, which has not been previously conceived nor demonstrated.

\section{Through-Floor Vital Sign Searching Concept}

The view of the proposed through-floor vital sign searching concept is plotted in Figure 1. To survey the trapped person, four UWB life-detection radars represented by pentagrams are activated and grouped in the wireless-netted way, and they are deployed and moved on a floor which forms a vital sign searching path in the 2D observation aperture (shown as the dark-green dotted arrow). Assuming some people with micromotions such as respiration or limb swing are trapped, these UWB life-detection radars are configured to detect these trapped persons. The working mode of the radar system is set as follows: first, the UWB life-detection radars are activated sequentially via remote network, avoiding mutual interference between radars at different observation points; and then, the radar echoes and results collected at different observation points need to be processed in accordance with the specially designed vital sign search procedure, avoiding wasting time dealing with clutter signals. In addition, when all observation points are sequentially selected and the transmission and reception of the mono-static radar is completed, this concept assumes that the trapped person retains the same/similar micro-motion during the vital sign searching process. The detailed design and key parameters of the UWB radar system can be found in our previous work [12]. The detailed parameters of the adopted UWB radar can be found in Table 1 .

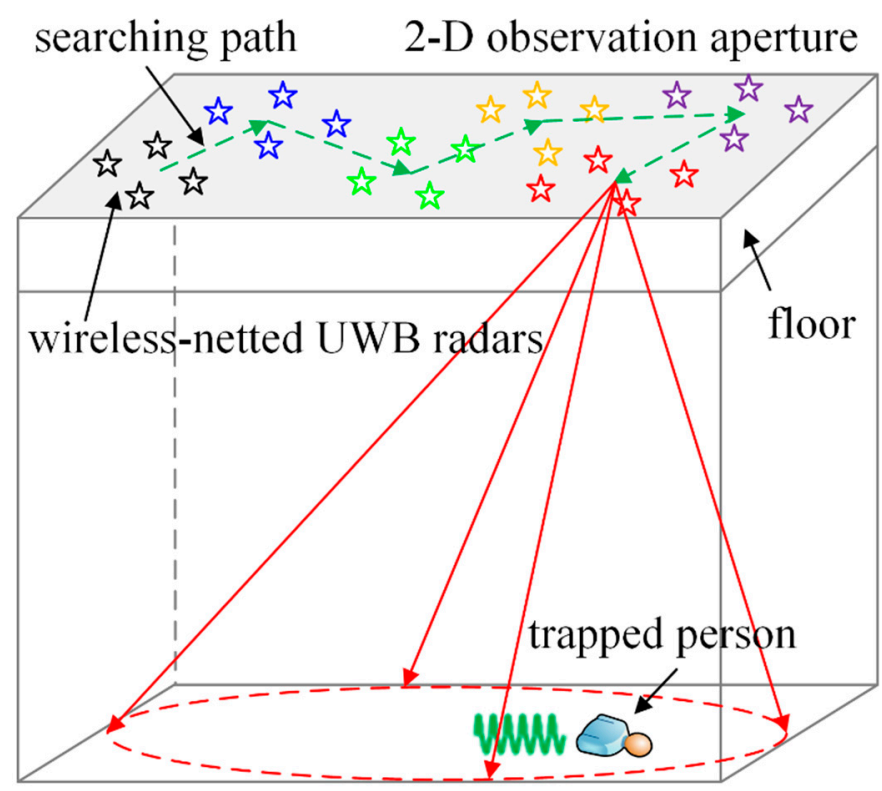

Figure 1. The concept for through-floor vital sign searching with wireless-netted UWB lifedetection radars. 
Table 1. Key parameters of the adopted wireless-netted UWB radar.

\begin{tabular}{cc}
\hline Parameters & Value \\
\hline Center frequency of UWB radar system & $500 \mathrm{MHz}$ \\
Bandwidth of UWB radar system & $500 \mathrm{MHz}$ \\
Equivalent sampling frequency (FS) & $20 \mathrm{GSPS}$ * \\
Real-time sampling frequency & $125 \mathrm{MSPS}{ }^{*}$ \\
Pulse Repeat Period (PRP) & $15.625 \mathrm{us}$ \\
Number of averaged values (NA) & 16 \\
Resolution of the ADC * & $16 \mathrm{bits}$ \\
Radar system scan rate & 25 scans $/ \mathrm{s}(\mathrm{Hz})$ \\
Sampling length (N) & 16,384 \\
Average power of radar signal & $<10 \mathrm{~mW}$ \\
Antenna type & Bow-tie Antenna \\
Beamwidth of the antenna & $60^{\circ}$ (E-plane) $\times 100^{\circ}$ (H- plane) \\
Polarization of the antenna & Linear polarization \\
Gain of the antenna & 3 dbi \\
\hline${ }^{*}$ GSPS: gigabit samples per second, MSPS: million samples per second, ADC: analog to digital converter.
\end{tabular}

\section{Through-Floor Vital Sign Search}

\subsection{Searching Path Design}

The wireless-netted UWB life-detection radars are deployed on the 2D floor to search for the trapped person, and the searching path should be designed accordingly. The searching path is composed of some specific deploying observation points where the radars are placed. Inspired by the design principle of 2D multiple-input multiple-output (MIMO) array topology, these deploying observation points can be designed and optimized. In our previous work [12], the 2D MIMO array topology is designed and presented, including $N_{T} \times N_{R}$ virtual elements in total. $N_{T}$ denotes the number of transmitters, and $N_{R}$ denotes the number of receivers. Here, the desired topology of the UWB life-detection radar deployment is constructed based on the optimizing way of the 2D MIMO array topology, and the observation points where the mono-static UWB life-detection radars are placed are equivalent to virtual elements. We employ $N_{T}=4$ transmitters, achieving a uniform-distribution characteristic in the 2D effective aperture, $N_{R}=N^{2}$ receivers are arranged inside the square formed by the transmitters. The $N_{T}=4$ transmitters are placed symmetrically at the four vertices of a square with a width of $\Delta_{A}$. The Cartesian coordinate is established and the location of four transmitters are as follows:

$$
\mathbf{r}_{\mathrm{T}}=\left\{\begin{array}{cc}
\left(\Delta_{A} / 2, \Delta_{A} / 2\right) & \left(\Delta_{A} / 2,-\Delta_{A} / 2\right) \\
\left(-\Delta_{A} / 2,-\Delta_{A} / 2\right) & \left(-\Delta_{A} / 2, \Delta_{A} / 2\right)
\end{array}\right\}
$$

Therefore, the selection range of all $N_{R}$ receivers are expressed as

$$
\mathbf{r}_{\mathrm{R}}=\left\{\left(x_{R_{n}}, y_{R_{n}}\right) \mid x_{R_{n}} \in \mathbf{s}, y_{R_{n}} \in \mathbf{s}, n=1, \ldots, N_{R}\right\}
$$

where $\mathbf{s}=\left[-\frac{N_{R}-1}{2} \cdot \frac{\Delta_{A}}{N_{R}}: \frac{\Delta_{A}}{N_{R}}: \frac{N_{R}-1}{2} \cdot \frac{\Delta_{A}}{N_{R}}\right]$, and when $i \neq j$, there will be $x_{R_{i}} \neq x_{R_{j}}$ and $y_{R_{i}} \neq y_{R_{j}}$.

Based on the configuration, observation points where the UWB life-detection radars are set are equivalent to the virtual elements, and the desired topology of the UWB lifedetection radar is achieved, optimizing the topology of the 2D MIMO array. The optimization method is summarized as follows.

- $\quad$ Step 1: Two basic principles:

(1) "Uniform distribution": All elements should uniformly cover over the 2D observation aperture with little redundancy, ensuring the equivalent line aperture of each angle cut can be as nearly uniform and periodic as possible. 
(2) "Little shadowing": In the equivalent line aperture of each angle, element shadowing, which is the elements overlap during the projection, should be as little as possible to obtain a unit weighted aperture.

- Step 2: Convert to MIMO topology design:

(1) According to the size of the area to be detected, set the size of the 2D observation aperture as $2 \Delta_{A} \times 2 \Delta_{A}$.

(2) Supposing $K^{2}$ observation points are required for 2D observation, in this manuscript, there are $K^{2}=36$ mono-static UWB life-detection radars to be arranged on the desired 2D observation aperture. Here, we set $K=6$.

(3) According to the concept of one-way 2D effective aperture corresponding to the 2D MIMO array, $N_{T} \times N_{R}=36$ virtual elements are taken as the observation points where the mono-static UWB life-detection radars are placed. Here, we set $N_{T}=4$ and $N_{R}=9$.

(4) Convert to the problem of $N_{T}=4$ and $N_{R}=92 \mathrm{D}$ MIMO array topology design.

- Step 3: Determine the transmitters' positions:

The $N_{T}=4$ transmitters are placed symmetrically at the four vertices of a square with a width of $\Delta_{A}$. The Cartesian coordinate is established and the location of four transmitters are as follows:

$$
\mathbf{r}_{\mathrm{T}}=\left\{\begin{array}{ll}
\left(x_{\mathrm{T} 1}, y_{\mathrm{T} 1}\right) & \left(x_{\mathrm{T} 2}, y_{\mathrm{T} 2}\right) \\
\left(x_{\mathrm{T} 3}, y_{\mathrm{T} 3}\right) & \left(x_{\mathrm{T} 4}, y_{\mathrm{T} 4}\right)
\end{array}\right\}=\left\{\begin{array}{cc}
\left(\Delta_{A} / 2, \Delta_{A} / 2\right) & \left(\Delta_{A} / 2,-\Delta_{A} / 2\right) \\
\left(-\Delta_{A} / 2,-\Delta_{A} / 2\right) & \left(-\Delta_{A} / 2, \Delta_{A} / 2\right)
\end{array}\right\}
$$

- Step 4: Determine the receivers' positions:

(1) The selection range of $N_{R}=9$ receivers are expressed as

$$
\mathbf{r}_{\mathrm{R}}=\left\{\left(x_{R_{n}}, y_{R_{n}}\right) \mid x_{R_{n}} \in \mathbf{s}, y_{R_{n}} \in \mathbf{s}, n=1, \ldots, 9\right\}
$$

where $\mathbf{s}=\left[-4 \frac{\Delta_{A}}{N_{R}}: \frac{\Delta_{A}}{N_{R}}: 4 \frac{\Delta_{A}}{N_{R}}\right]$, and when $i \neq j$, there will be $x_{R_{i}} \neq x_{R_{j}}$ and $y_{R_{i}} \neq y_{R_{j}}$.

(2) The effective virtual elements can be expressed as $\mathbf{r}_{\text {eff }}$, including 36 positions.

$$
\begin{aligned}
\mathbf{r}_{\mathrm{eff}}=\mathbf{r}_{\mathrm{T}}+\mathbf{r}_{\mathrm{R}} & =\left\{\begin{array}{llll}
\left(x_{\mathrm{T} 1}+x_{\mathrm{R} 1}, y_{\mathrm{T} 1}+y_{\mathrm{R} 1}\right) & \left(x_{\mathrm{T} 2}+x_{\mathrm{R} 1}, y_{\mathrm{T} 2}+y_{\mathrm{R} 1}\right) & \left(x_{\mathrm{T} 3}+x_{\mathrm{R} 1}, y_{\mathrm{T} 3}+y_{\mathrm{R} 1}\right) & \left(x_{\mathrm{T} 4}+x_{\mathrm{R} 1}, y_{\mathrm{T} 4}+y_{\mathrm{R} 1}\right) \\
\left(x_{\mathrm{T} 1}+x_{\mathrm{R} 2}, y_{\mathrm{T} 1}+y_{\mathrm{R} 2}\right) & \left(x_{\mathrm{T} 2}+x_{\mathrm{R} 2}, y_{\mathrm{T} 2}+y_{\mathrm{R} 2}\right) & \left(x_{\mathrm{T} 3}+x_{\mathrm{R} 2}, y_{\mathrm{T} 3}+y_{\mathrm{R} 2}\right) & \left(x_{\mathrm{T} 4}+x_{\mathrm{R} 2}, y_{\mathrm{T} 4}+y_{\mathrm{R} 2}\right) \\
\left(x_{\mathrm{T} 1}+x_{\mathrm{R} 3}, y_{\mathrm{T} 1}+y_{\mathrm{R} 3}\right) & \left(x_{\mathrm{T} 2}+x_{\mathrm{R} 3}, y_{\mathrm{T} 2}+y_{\mathrm{R} 3}\right) & \left(x_{\mathrm{T} 3}+x_{\mathrm{R} 3}, y_{\mathrm{T} 3}+y_{\mathrm{R} 3}\right) & \left(x_{\mathrm{T} 4}+x_{\mathrm{R} 3}, y_{\mathrm{T} 4}+y_{\mathrm{R} 3}\right) \\
\left(x_{\mathrm{T} 1}+x_{\mathrm{R} 4}, y_{\mathrm{T} 1}+y_{\mathrm{R} 4}\right) & \left(x_{\mathrm{T} 2}+x_{\mathrm{R} 4}, y_{\mathrm{T} 2}+y_{\mathrm{R} 4}\right) & \left(x_{\mathrm{T} 3}+x_{\mathrm{R} 4}, y_{\mathrm{T} 3}+y_{\mathrm{R} 4}\right) & \left(x_{\mathrm{T} 4}+x_{\mathrm{R} 4}, y_{\mathrm{T} 4}+y_{\mathrm{R} 4}\right) \\
\left(x_{\mathrm{T} 1}+x_{\mathrm{R} 5}, y_{\mathrm{T} 1}+y_{\mathrm{R} 5}\right) & \left(x_{\mathrm{T} 2}+x_{\mathrm{R} 5}, y_{\mathrm{T} 2}+y_{\mathrm{R} 5}\right) & \left(x_{\mathrm{T} 3}+x_{\mathrm{R} 5}, y_{\mathrm{T} 3}+y_{\mathrm{R} 5}\right) & \left(x_{\mathrm{T} 4}+x_{\mathrm{R} 5}, y_{\mathrm{T} 4}+y_{\mathrm{R} 5}\right) \\
\left(x_{\mathrm{T} 1}+x_{\mathrm{R} 6}, y_{\mathrm{T} 1}+y_{\mathrm{R} 6}\right) & \left(x_{\mathrm{T} 2}+x_{\mathrm{R} 6}, y_{\mathrm{T} 2}+y_{\mathrm{R} 6}\right) & \left(x_{\mathrm{T} 3}+x_{\mathrm{R} 6}, y_{\mathrm{T} 3}+y_{\mathrm{R} 6}\right) & \left(x_{\mathrm{T} 4}+x_{\mathrm{R} 6}, y_{\mathrm{T} 4}+y_{\mathrm{R} 6}\right) \\
\left(x_{\mathrm{T} 1}+x_{\mathrm{R} 7}, y_{\mathrm{T} 1}+y_{\mathrm{R} 7}\right) & \left(x_{\mathrm{T} 2}+x_{\mathrm{R}}, y_{\mathrm{T} 2}+y_{\mathrm{R} 7}\right) & \left(x_{\mathrm{T} 3}+x_{\mathrm{R} 7}, y_{\mathrm{T} 3}+y_{\mathrm{R} 7}\right) & \left(x_{\mathrm{T} 4}+x_{\mathrm{R} 7}, y_{\mathrm{T} 4}+y_{\mathrm{R} 7}\right) \\
\left(x_{\mathrm{T} 1}+x_{\mathrm{R} 8}, y_{\mathrm{T} 1}+y_{\mathrm{R}}\right) & \left(x_{\mathrm{T} 2}+x_{\mathrm{R} 8}, y_{\mathrm{T} 2}+y_{\mathrm{R} 8}\right) & \left(x_{\mathrm{T} 3}+x_{\mathrm{R} 8}, y_{\mathrm{T} 3}+y_{\mathrm{R} 8}\right) & \left(x_{\mathrm{T} 4}+x_{\mathrm{R} 8}, y_{\mathrm{T} 4}+y_{\mathrm{R} 8}\right) \\
\left(x_{\mathrm{T} 1}+x_{\mathrm{R} 9}, y_{\mathrm{T} 1}+y_{\mathrm{R} 9}\right) & \left(x_{\mathrm{T} 2}+x_{\mathrm{R} 9}, y_{\mathrm{T} 2}+y_{\mathrm{R} 9}\right) & \left(x_{\mathrm{T} 3}+x_{\mathrm{R} 9}, y_{\mathrm{T} 3}+y_{\mathrm{R} 9}\right) & \left(x_{\mathrm{T} 4}+x_{\mathrm{R} 9}, y_{\mathrm{T} 4}+y_{\mathrm{R} 9}\right)
\end{array}\right\}
\end{aligned}
$$

(3) Divide the 2D observation aperture $\left(2 \Delta_{A} \times 2 \Delta_{A}\right)$ into $K \times K=6 \times 6$ grids, as shown by the red grids in Figure 2. Meanwhile, each grid must contain only one effective virtual element from $\mathbf{r}_{\text {eff }}$, which is determined by the "two basic principles" in Step 1. 


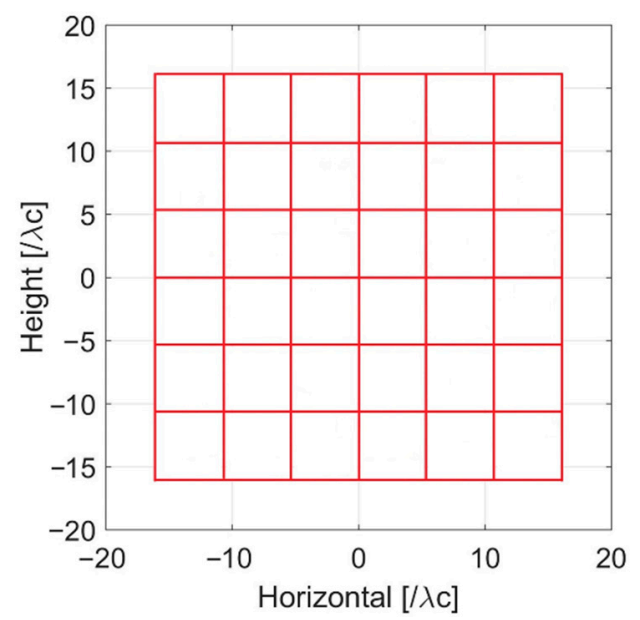

Figure 2. The $2 \mathrm{D}$ observation aperture divided into $6 \times 6$ grids.

(4) Evenly divide each grid into $\sqrt{N_{R}} \times \sqrt{N_{R}}=3 \times 3$ positions, as shown by the black circles in Figure 3. Thus, there are $(3 \times 6) \times(3 \times 6)=324$ optional positions to place effective virtual elements.

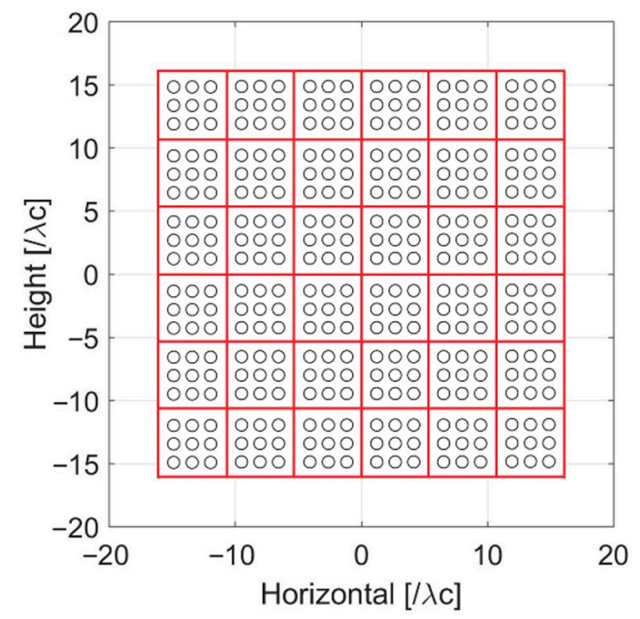

Figure 3. There are 324 optional positions to place effective virtual elements.

(5) According to Step 4.1, by traversing all possible $\mathbf{r}_{R}$, the 36 effective virtual elements from the resultant $\mathbf{r}_{\text {eff }}$ would distribute randomly into 324 optional positions. We define a scoring rule: (i) Give the grid zero score when there are no virtual elements in it; (ii) otherwise, give the grid one score; (iii) the highest total score of all grids equal to 36 . The 36 score indicates that the corresponding traversing result of $\mathbf{r}_{\mathrm{R}}$ is satisfied with the "two basic principles" in Step 1. For example, as shown in Figure 4, by optimizing the MIMO array topology, the effective aperture with different scores can be obtained. In Figure $4 a, b$, the score is 36 and the corresponding topology-I is available. In Figure 4c,d, the score is 28 and the corresponding topology-II is unavailable. 


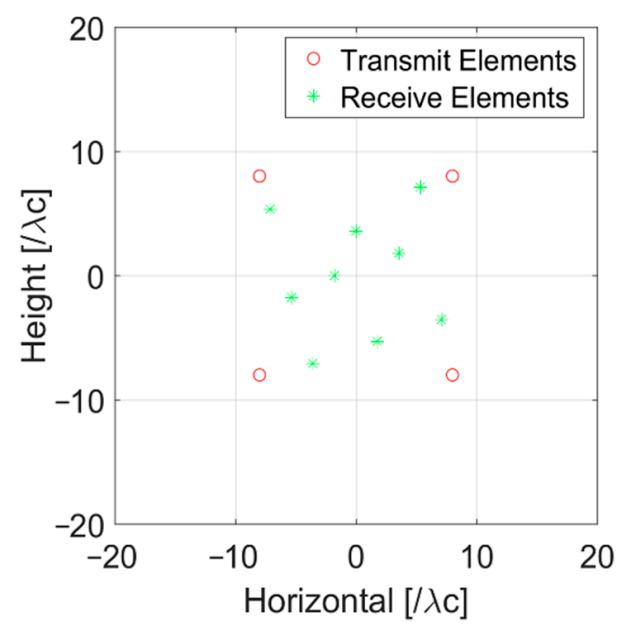

(a)

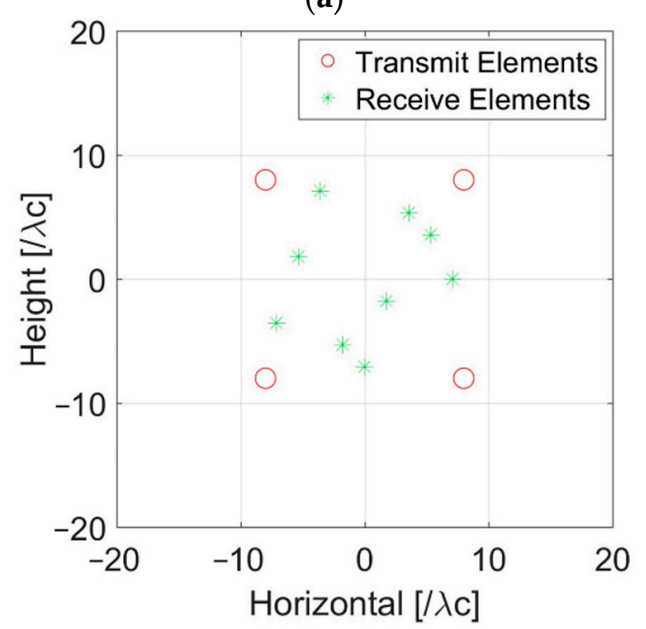

(c)

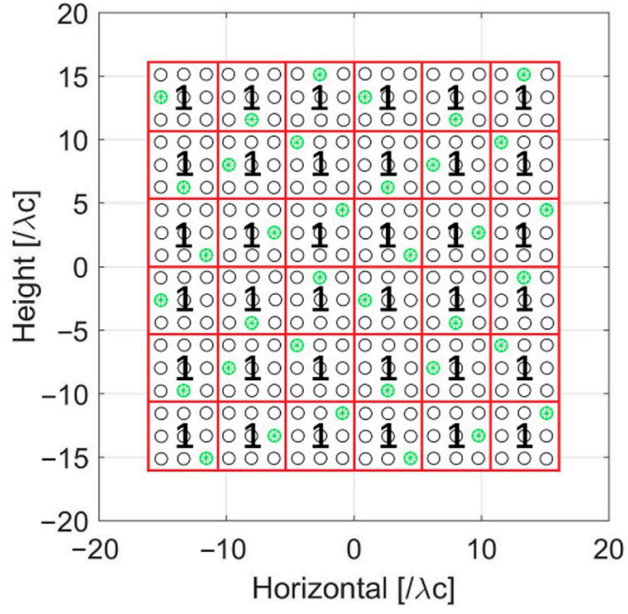

(b)

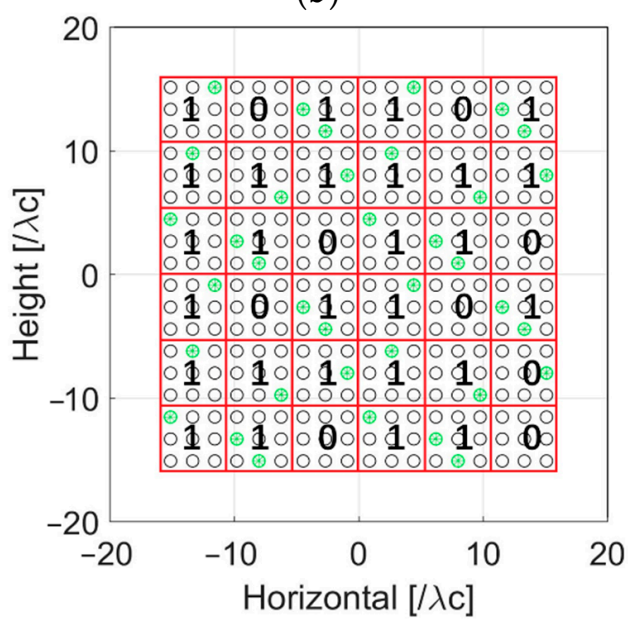

(d)

Figure 4. Different MIMO array topologies and effective aperture with different scores. (a) Topology-I; (b) effective aperture with 36 score; (c) Topology-II; (d) effective aperture with 28 score.

\subsection{Vital Sign Searching Procedure}

The schematic of the vital sign searching procedure is described in Figure 5 and is summarized as follows.

(1) Record the four wireless-netted radars' spatial locations $\left(x_{1}, y_{1}, z_{1}\right),\left(x_{2}, y_{2}, z_{2}\right)$, $\left(x_{3}, y_{3}, z_{3}\right)$, and $\left(x_{4}, y_{4}, z_{4}\right)$. The corresponding detection distances for the trapped person are represented by $R_{1}, R_{2}, R_{3}$, and $R_{4}$. Construct the detection distance vector $\left(R_{1}, R_{2}, R_{3}, R_{4}\right)$.

(2) Invalid value elimination and classification. If $R_{i} \neq 0, i \in\{1,2,3,4\}$, the value of $R_{i}$ is valid. Judge the number of effective values in the detection distance vector; the number of effective values might be $4,3,2,1,0$.

(3) Valid value number $=4$. Choose three valid values each time and check whether the three observation points corresponding to the three valid values satisfy the "triangle composition condition". If the three observation points fail, we reselect three valid values from these four valid values. Otherwise, the three detection points can be used to calculate the spatial location of the target by using "noncoherent positioning" method. Then, repeat this procedure four times. For through-floor case, the "noncoherent positioning" equation set is

$$
\left\{\begin{array}{l}
\left(x-x_{i}\right)^{2}+\left(y-y_{i}\right)^{2}+z^{2}=R_{i}^{2} \\
\left(x-x_{j}\right)^{2}+\left(y-y_{j}\right)^{2}+z^{2}=R_{j}^{2} \\
\left(x-x_{k}\right)^{2}+\left(y-y_{k}\right)^{2}+z^{2}=R_{k}^{2}
\end{array}\right.
$$

where $i, j, k \in\{1,2,3,4\}$. 
(4) Valid value number $=3$. Use the "noncoherent positioning" method to obtain the spatial location of the target and judge the existence of the solution. If the solution with $z<0$ exists, then it is the spatial location of the target. Otherwise, the "triangle composition condition" should be used to judge which observation point is improper and eliminate it by setting its detection distance to zero. The improper observation point always has the largest detection distance. Then, rejudge the number of the valid values.

(5) Valid value number $=2$. According to the "triangle composition condition", when the distances $D_{i, j},(i, j \in\{1,2,3,4\})$ between the two observation points and the two detection distances $R_{i}, R_{j},(i, j \in\{1,2,3,4\})$ should satisfy the condition of $R_{i}+R_{j}>D_{i, j}$. The detection distance $R_{i},(i \in\{1,2,3,4\})$, by processing the echoes obtained from the $i$-th UWB radar, is taken as the 1D solution. Otherwise, the observation point corresponding to the smaller value of $R_{i}, R_{j}$ is reserved as the $1 \mathrm{D}$ solution. Further, we can select two or three new observation points to reconstruct four observation points and repeat the above procedure.

(6) Valid value number $=1$. There is only the 1D solution. Further, we can keep the corresponding observation point, select three new observation points and repeat the above procedure.

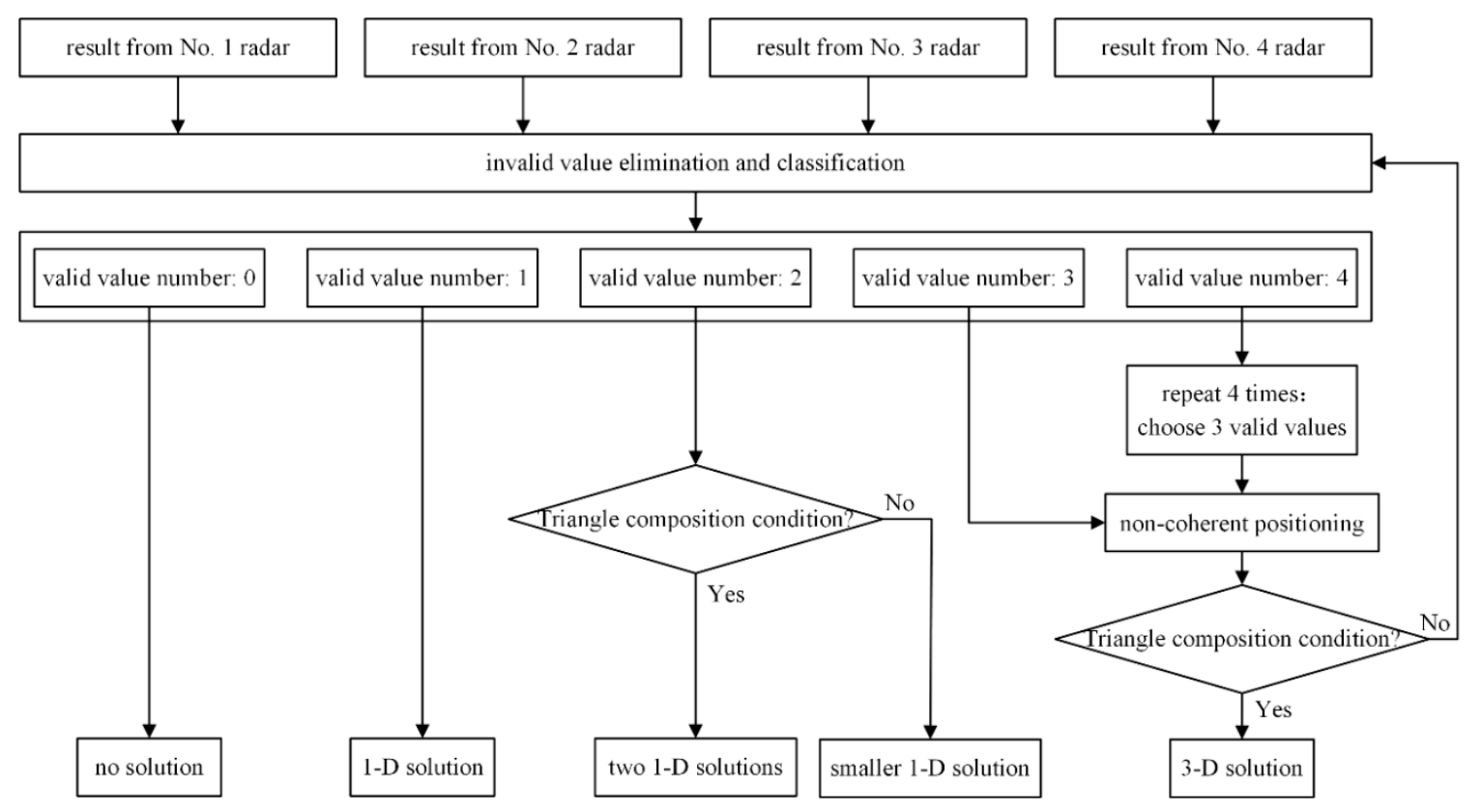

Figure 5. The vital sign searching procedure.

\section{Results}

\subsection{Simulated Result}

In this section, the simulation is performed to illustrate the proposed vital sign searching concept in Section 3. According to the abovementioned "searching path design" method, as shown in Figure 6a, the 2D observation aperture is divided into $6 \times 6$ grids and there is one observation point in each grid (marked by green star-circle). Then, in Figure $6 b$, four wireless-netted UWB life-detection radars (marked by four red pentagrams) can be deployed around one observation point. Note that only one observation point should be selected during one measurement. The observation point can be selected randomly for the first measurement. Subsequently, the observation point for the next measurement should be determined by the vital sign searching procedure. Finally, after several measurements, the vital sign of the trapped person can be searched, and the spatial location can be obtained. As shown in Figure 6c, the vital sign searching path is composed of four observation points, and the vital sign of the trapped person (marked by the black diamond) is searched nearby 
the fourth observation point. The position of the simulated trapped person is set as $(3.75 \mathrm{~m}$, $-3.75 \mathrm{~m}, 3.75 \mathrm{~m})$.

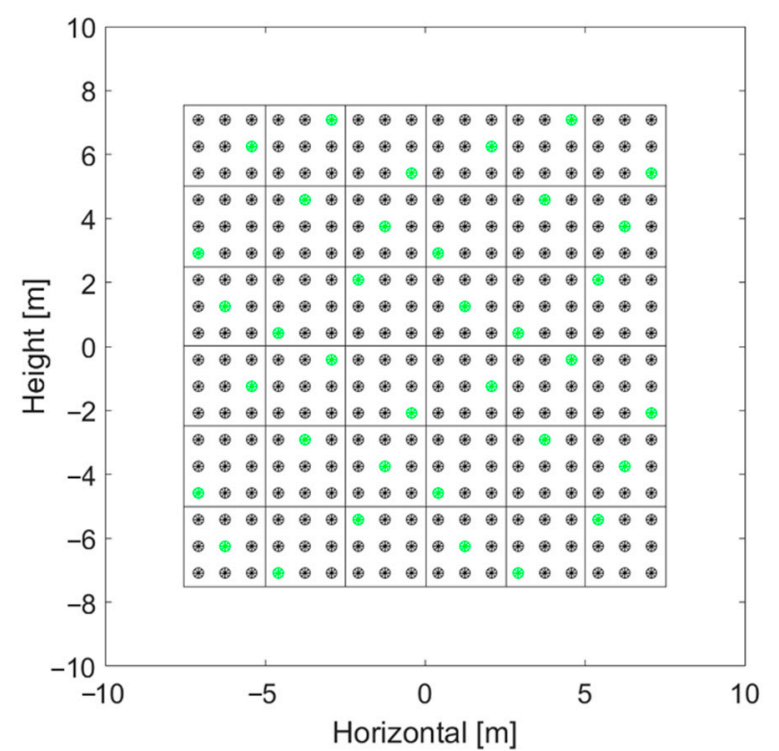

(a)

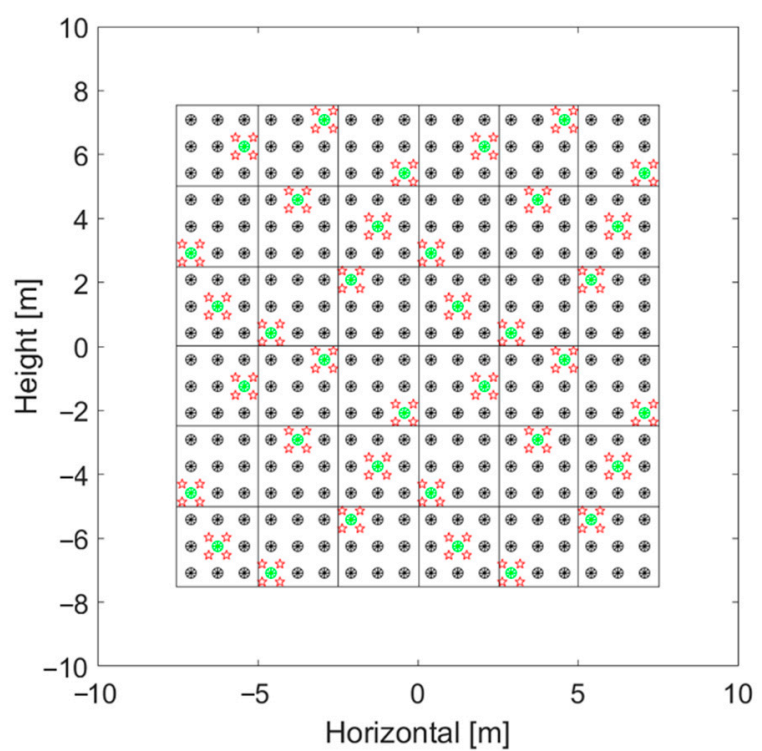

(b)

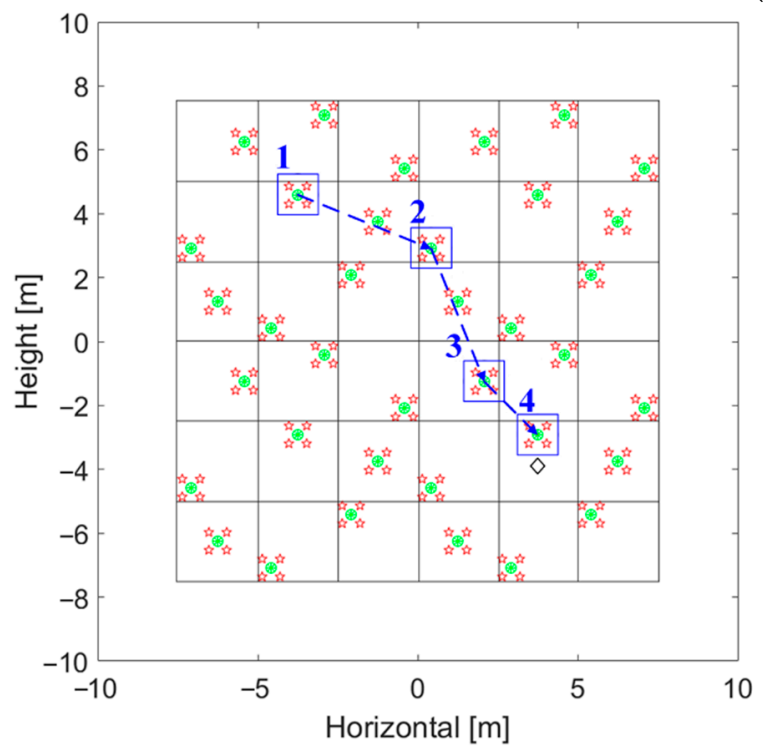

(c)

Figure 6. The schematic of searching a trapped person along a searching path. (a) The available observation points in the 2D observation aperture; (b) the wireless-netted radars are deployed around the observation point; (c) the vital sign searching path.

The detailed results in such a vital sign searching process are listed in Table 2. The first observation point is "observation point $1(-3.75 \mathrm{~m}, 4.583 \mathrm{~m}, 0 \mathrm{~m})$ ", and the detection results obtained from no. 1 no. 4 radars around the observation point are $13.4 \mathrm{~m}, 12.61 \mathrm{~m}, 13.89 \mathrm{~m}$, and $12.28 \mathrm{~m}$, respectively. The corresponding output indicates the next observation point might be "observation point $2(0.42 \mathrm{~m}, 2.9 \mathrm{~m}, 0 \mathrm{~m})$ ". Subsequently, the final observation point might be "observation point $4(3.75 \mathrm{~m},-2.917 \mathrm{~m}, 0 \mathrm{~m})$ ", and the output result of $(3.72 \mathrm{~m},-3.75 \mathrm{~m}, 3.765 \mathrm{~m})$ should be the spatial location of the vital sign of the trapped person, which is in accordance with the simulated setup. This process can also be illustrated by Figure 7 . The red dotted arrow points to the next possible observation point, and the light-blue dotted frame indicates the possible spatial location of the vital sign. Finally, the vital sign searching path composed of four observation points can be formed (marked by 
the blue dotted arrows). The judgment rule can be summarized as: (1) the virtual elements located in this red dot-line frame should be considered as the next possible observation point; (2) one virtual element is selected as the next observation point which is nearest to one solution represented by a red dot; (3) the searching process represented by the blue dot-line arrow is completed when the rectangular suspected area is small enough and contains only one same virtual element by at least two iterations.
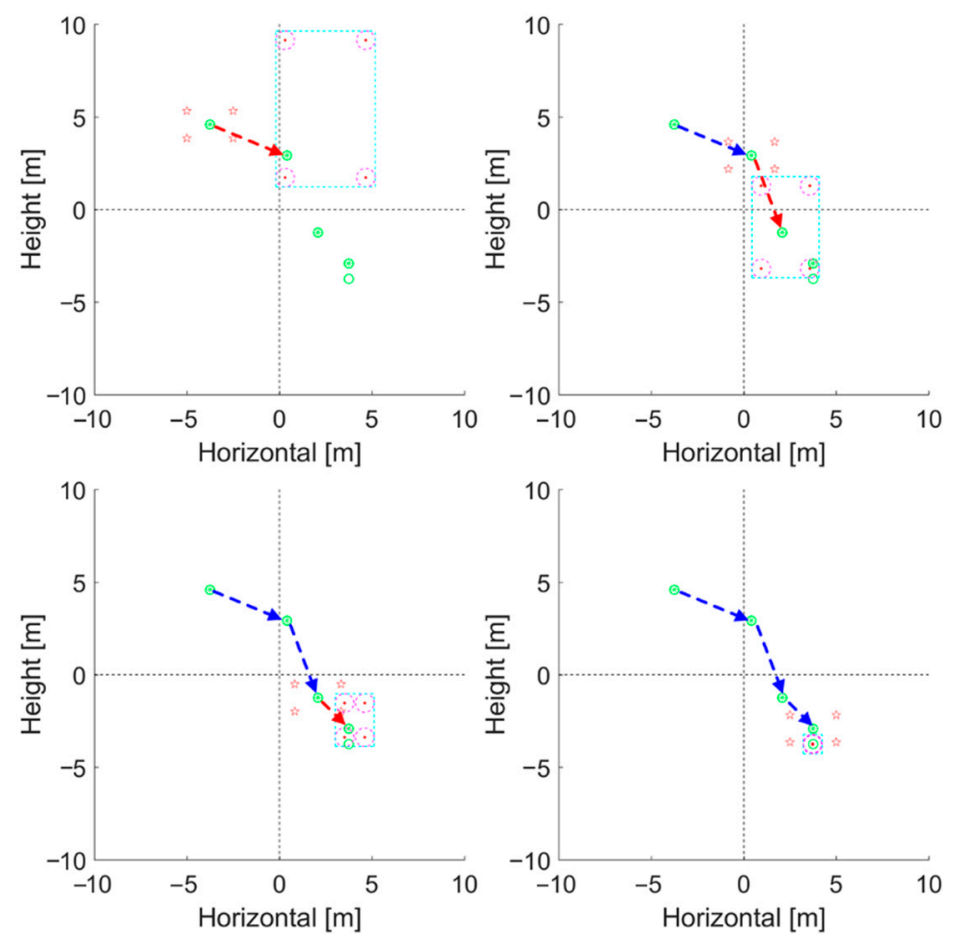

Figure 7. The simulated result of searching for a trapped person along a searching path.

Table 2. The simulated result for the vital sign searching path.

\begin{tabular}{ccccc}
\hline Searching Path & Observation Point 1 & Observation Point 2 & Observation Point 3 & Observation Point 4 \\
\hline & $(-3.75 \mathrm{~m}, 4.58 \mathrm{~m}, 0 \mathrm{~m})$ & $(0.42 \mathrm{~m}, 2.92 \mathrm{~m}, 0 \mathrm{~m})$ & $(2.08 \mathrm{~m},-1.25 \mathrm{~m}, 0 \mathrm{~m})$ & $(3.75 \mathrm{~m},-2.92 \mathrm{~m}, 0 \mathrm{~m})$ \\
\hline No. 1 radar result & $13.4 \mathrm{~m}$ & $9.69 \mathrm{~m}$ & $5.8 \mathrm{~m}$ & $4.26 \mathrm{~m}$ \\
No. 2 radar result & $12.61 \mathrm{~m}$ & $8.84 \mathrm{~m}$ & $5.15 \mathrm{~m}$ & $4.27 \mathrm{~m}$ \\
No. 3 radar result & $13.89 \mathrm{~m}$ & $8.71 \mathrm{~m}$ & $5.73 \mathrm{~m}$ & $3.96 \mathrm{~m}$ \\
No. 4 radar result & $12.28 \mathrm{~m}$ & $8.56 \mathrm{~m}$ & $3.5 \mathrm{~m}$ & $3.98 \mathrm{~m}$ \\
& $(0.31 \mathrm{~m}, 1.72 \mathrm{~m}, 11.76 \mathrm{~m})$ & $(0.94 \mathrm{~m},-3.19 \mathrm{~m}, 6.63 \mathrm{~m})$ & $(3.52 \mathrm{~m},-3.38 \mathrm{~m}, 4.27 \mathrm{~m})$ & \\
Output & $(0.31 \mathrm{~m}, 9.14 \mathrm{~m}, 11.69 \mathrm{~m})$ & $(0.94 \mathrm{~m}, 1.28 \mathrm{~m}, 8.48 \mathrm{~m})$ & $(3.52 \mathrm{~m},-1.53 \mathrm{~m}, 5.04 \mathrm{~m})$ & $(3.72 \mathrm{~m},-3.75 \mathrm{~m}, 3.77 \mathrm{~m})$ \\
& $(4.67 \mathrm{~m}, 1.72 \mathrm{~m}, 9.73 \mathrm{~m})$ & $(3.57 \mathrm{~m},-3.19 \mathrm{~m}, 5.26 \mathrm{~m})$ & $(4.61 \mathrm{~m},-3.38 \mathrm{~m}, 4.08 \mathrm{~m})$ & \\
\hline
\end{tabular}

\subsection{Experimental Results}

As shown in Figure 8a, the performance of the proposed vital sign searching based on different observation is verified and evaluated using a through-floor experiment, in which four wireless-netted connected UWB life-detection radars are adopted. The size of the floor is $3.2 \mathrm{~m} \times 4 \mathrm{~m}$, with one pipeline inside. The position of the simulated trapped person is marked by the yellow diamond in Figure 8c. One human test subject lay on the ground with weak respiration, acting as a trapped person (see Figure $8 b$ ), and the radar echoes are obtained from 10 time detections (see Figure 8c). To confirm the spatial location of the human test subject, we can select any four time detections to form an available vital sign searching path, which is marked by the green arrow in Figure 8c.

The detailed experimental results in such a vital sign searching process are listed in Table 3. Firstly, the detection results from the No. 7, No. 8, No. 9, and No. 10 radars are 
performed to calculate the next possible observation point. The corresponding observation point 1 is $(0.8 \mathrm{~m}, 1.2 \mathrm{~m}, 3.75 \mathrm{~m})$. The output result of $(-0.38 \mathrm{~m}, 0.02 \mathrm{~m}, 4.19 \mathrm{~m})$ indicates that the next observation point might be "observation point $2(-0.4 \mathrm{~m}, 0 \mathrm{~m}, 3.75 \mathrm{~m})$ ". Subsequently, the final observation point might be "observation point $3(-0.4 \mathrm{~m}, 0.8 \mathrm{~m}$, $3.75 \mathrm{~m})^{\prime \prime}$, and the output result of $(-0.47 \mathrm{~m}, 0.79 \mathrm{~m}, 3.76 \mathrm{~m})$ should be the spatial location of the vital sign of the trapped person, which is in accordance with the experimental setup. Similar to the simulation, this process can also be illustrated by Figure 9. The vital sign searching path composed of three observation points can be formed (marked by the green arrows).

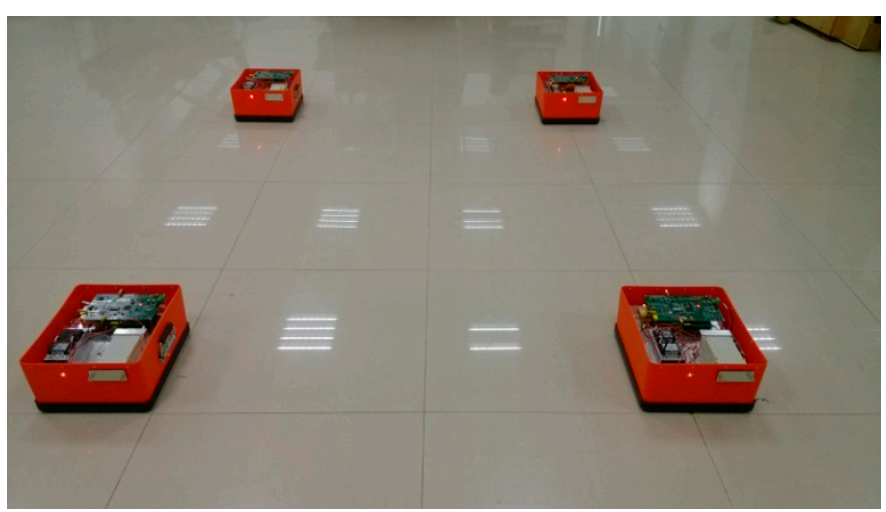

(a)

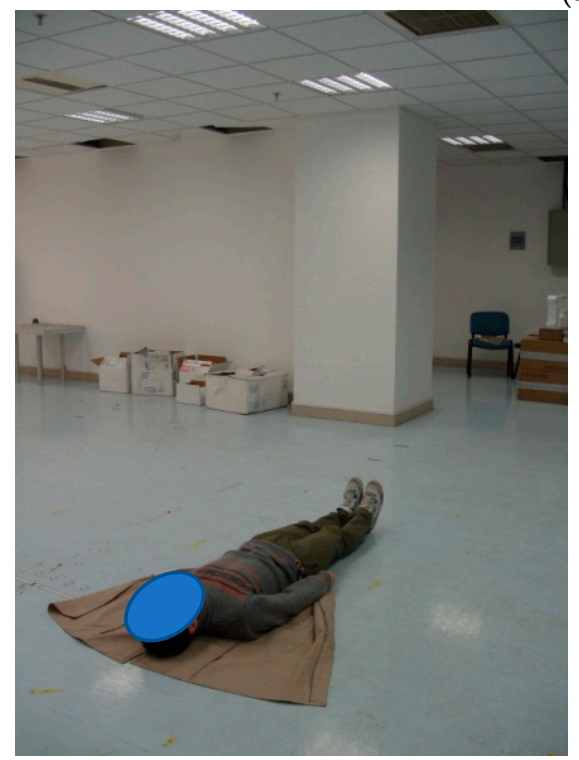

(b)

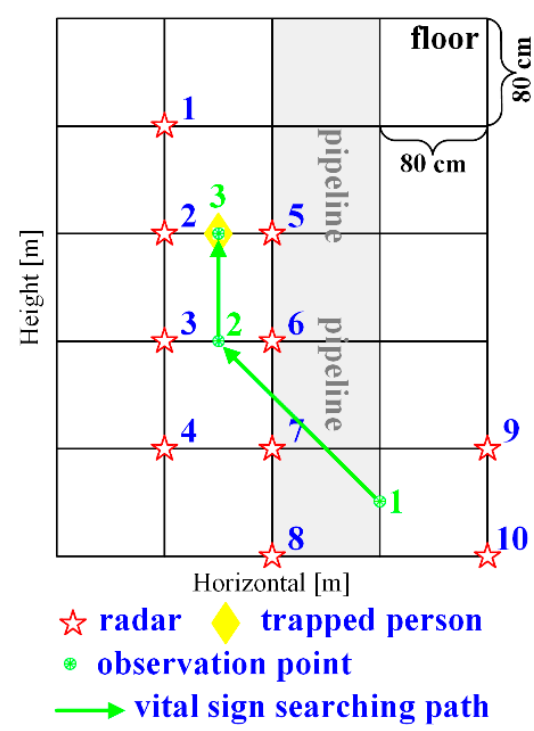

(c)

Figure 8. The experimental setup. (a) The four wireless-netted UWB life-detection radars deployed on a floor; (b) the human test subject acting as a trapped person; (c) the top view of the $2 \mathrm{D}$ observation aperture and the vital sign searching path. 


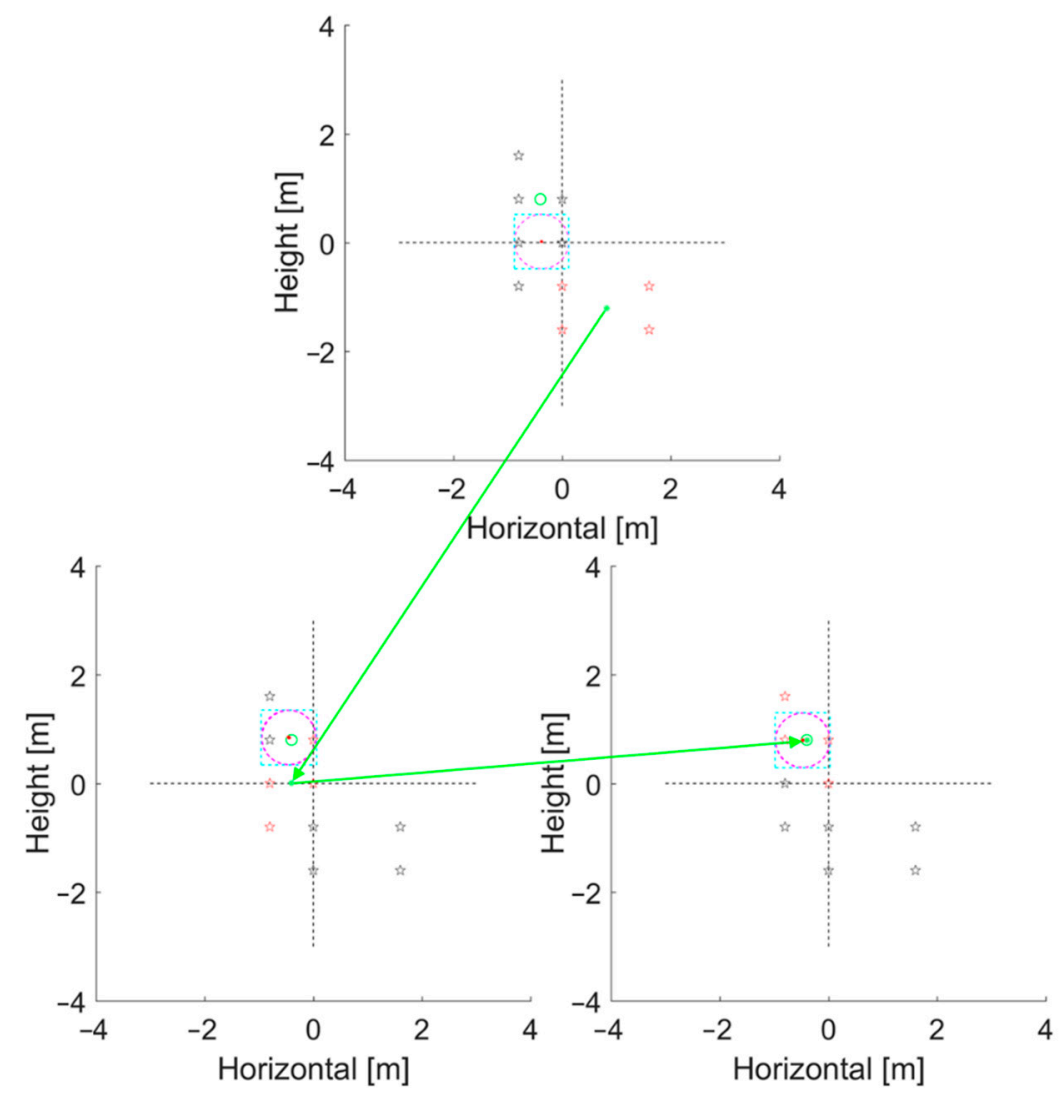

Figure 9. The experimental result of searching for a trapped person along a searching path.

Table 3. The experimental result for the vital sign searching path.

\begin{tabular}{cccc}
\hline Searching Path & Observation Point 1 & Observation Point 2 & Observation Point 3 \\
\hline & $(0.8 \mathrm{~m}, 1.2 \mathrm{~m}, 3.75 \mathrm{~m})$ & $(-0.4 \mathrm{~m}, 0 \mathrm{~m}, 3.75 \mathrm{~m})$ & $(-0.4 \mathrm{~m}, 0.8 \mathrm{~m}, 3.75 \mathrm{~m})$ \\
\hline No.1 radar result & & & 3.86 \\
No.2 radar result & & 3.87 & 3.77 \\
No.3 radar result & & 4.12 & 3.79 \\
No.4 radar result & 4.29 & 3.88 & 3.87 \\
No.5 radar result & 4.51 & & \\
No.6 radar result & 0 & & \\
No.7 radar result & 4.91 & & \\
No.8 radar result & & & \\
No.9 radar result & & & \\
No.10 radar result & $(-0.38 \mathrm{~m}, 0.02 \mathrm{~m}, 4.19 \mathrm{~m})$ & $(-0.45 \mathrm{~m}, 0.85 \mathrm{~m}, 3.76 \mathrm{~m})$ & \\
Output & & &
\end{tabular}

\section{Conclusions}

A novel multiple-observation-points-based vital sign searching concept using wirelessnetted UWB life-detection radars has been developed for the searching and rescuing of through-floor trapped persons. Note that the echoes and detection results collected from different observation points need to be processed following an associated means of vital sign search procedure, and the selecting and optimization of the searching path are presented. By adopting the proposed vital sign searching procedure, the radar system is able to locate the spatial position of the trapped person by way of associating the diversity of detection results from different observation points. Furthermore, simulation and experiment were conducted, showing the validation of the proposed vital sign searching path concept. 
Author Contributions: The research was performed by the authors as follows: conceptualization, Z.X. and S.W.; methodology, Z.X. and S.W.; software, S.W. and X.L.; validation, Z.X. and S.W.; formal analysis, S.W. and X.L.; investigation, Z.X. and H.S.; resources, S.J. and T.P.; data curation, X.L. and S.W.; writing-original draft preparation, X.L. and S.W.; writing-review and editing, X.L. and Z.X.; visualization, X.L. and S.W.; supervision, S.J. and T.P.; project administration, Z.X. and H.S.; funding acquisition, Z.X. All authors have read and agreed to the published version of the manuscript.

Funding: This research work was supported by Innovation Funds of Equipment Pre-Research under Grant JZX7Y20190253040601 and JZX7Y20190253036001. The authors wish to express their gratitude to the editor and the anonymous reviewers.

Conflicts of Interest: The authors declare no conflict of interest.

\section{References}

1. Liu, L.; Liu, Z.; Barrowes, B. Through-wall bio-radiolocation with UWB impulse radar-Observation, simulation and signal extraction. IEEE J. Sel. Top. Appl. Earth Observ. Remote Sens. 2011, 4, 791-798. [CrossRef]

2. Schleicher, B.; Nasr, I.; Trasser, A.; Schumacher, H. IR-UWB radar demonstrator for ultra-fine movement detection and vital-sign monitoring. IEEE Trans. Microw. Theory Technol. 2013, 61, 2076-2085. [CrossRef]

3. Li, Z.; Li, W.; Lv, H.; Zhang, Y.; Jing, X.; Wang, J. A novel method for respiration-like clutter cancellation in life detection by dual-frequency IR-UWB radar. IEEE Trans. Microw. Theory Technol. 2013, 61, 2086-2092. [CrossRef]

4. Jing, L.; Liu, L.; Zeng, Z.; Liu, F. Advanced signal processing for vital sign extraction with applications in UWB radar detection of trapped victims in complex environments. IEEE J. Sel. Top. Appl. Earth Observ. Remote Sens. 2014, 7, 783-791.

5. Liu, L.; Liu, Z.; Xie, H.; Barrowes, B.; Bagtzoglou, A.C. Numerical simulation of UWB impulse radar vital sign detection at an earthquake disaster site. Ad Hoc Netw. 2014, 13, 34-41. [CrossRef]

6. Tu, J.; Hwang, T.; Lin, J. Respiration rate measurement under 1-D body motion using single continuous-wave doppler radar vital sign detection system. IEEE Trans. Microw. Theory Technol. 2016, 64, 1937-1946. [CrossRef]

7. Wang, K.; Zeng, Z.; Sun, J. Through-wall detection of the moving paths and vital signs of human beings. IEEE Geosci. Remote Sens. Lett. 2019, 16, 717-721. [CrossRef]

8. Mercuri, M.; Lorato, I.R.; Liu, Y.; Wieringa, F.; Hoof, C.V.; Torfs, T. Vital-sign monitoring and spatial tracking of multiple people using a contactless radar-based sensor. Nat. Electron. 2019, 2, 252-262. [CrossRef]

9. Lv, H.; Qi, F.; Zhang, Y.; Jiao, T.; Liang, F.; Li, Z.; Wang, J. Improved detection of human respiration using data fusion based on a multistatic UWB radar. Remote Sens. 2016, 8, 773. [CrossRef]

10. Wu, S.; Yao, S.; Liu, W.; Tan, K.; Xia, Z.; Meng, S.; Chen, J.; Fang, G.; Yin, H. Study on a novel UWB linear array human respiration model and detection method. IEEE J. Sel. Top. Appl. Earth Observ. Remote Sens. 2016, 9, 125-140. [CrossRef]

11. Jia, Y.; Guo, Y.; Yan, C.; Sheng, H.; Cui, G.; Zhong, X. Detection and localization for multiple stationary human targets based on cross-correlation of dual-station SFCW radars. Remote Sens. 2019, 11, 1428. [CrossRef]

12. Yan, K.; Wu, S.; Ye, S.; Fang, G. A novel wireless-netted UWB life-detection radar system for quasi-static person sensing. Appl. Sci. 2021, 11, 424. [CrossRef]

13. Debes, C.; Amin, M.G.; Zoubir, A.M. Target detection in single- and multiple-view through-the-wall radar imaging. IEEE Trans. Geosci. Remote Sens. 2009, 47, 1349-1361. [CrossRef]

14. Geng, Z. Evolution of netted radar systems. IEEE Access 2020, 8, 124961-124977. [CrossRef] 